\title{
A CONDITION IN INVARIANT FORM FOR A NET
} WITHOUT DETOURS*

BY ROBIN ROBINSON

Suppose that on a surface whose linear element is given by

$$
d s^{2}=E d u^{2}+2 F d u d v+G d v^{2}, \quad D^{2}=E G-F^{2} \neq 0,
$$

we have a system of curves, one and only one curve of each family passing through each point of that region of the surface under consideration, and distances along these curves being measured positively in a direction preassigned for each family. If this system of curves possesses the property that the distance between any two points of the region, measured along curves of the system according to the preassigned laws of orientation, is independent of the path chosen, then the system is called a net without detours (Kurvennetz ohne Umwege).

A net without detours being given, the distance along curves of the net from a fixed point $O$ to a variable point $P:(u, v)$ must be a function of position only. If the function $f(u, v)$ is given, and we wish to find the net without detours corresponding to it, we must have for the curves of the net $d f^{2}=d s^{2}$, or,

$$
\left(f_{u} d u+f_{v} d v\right)^{2}=E d u^{2}+2 F d u d v+G d v^{2},
$$

or,

$$
\left(E-f_{u}^{2}\right) d u^{2}+2\left(F-f_{u} f_{v}\right) d u d v+\left(G-f_{v}^{2}\right) d v^{2}=0 .
$$

This differential equation defines the net without detours corresponding to $f(u, v)$.

If a family of curves $\phi(u, v)=$ const. is to be one family of a net without detours for a given distance function $f(u, v)$, then $\left(\phi_{u} d u+\phi_{v} d v\right)$ must be a factor of the left side of (1), so that their Sylvester eliminant must vanish:

$$
\left|\begin{array}{ccc}
E-f_{u}^{2} & 2\left(F-f_{u} f_{v}\right) & G-f_{v}^{2} \\
\phi_{u} & \phi_{v} & 0 \\
0 & \phi_{u} & \phi_{v}
\end{array}\right|=0,
$$

\footnotetext{
* Presented to the Society, September 3, 1936.
} 


$$
\begin{aligned}
\left(\phi_{v} f_{u}-\phi_{u} f_{v}\right)^{2} & =E \phi_{v}{ }^{2}-2 F \phi_{u} \phi_{v}+G \phi_{u}{ }^{2} \\
{[J(f, \phi)]^{2} } & =D^{2} \Delta_{1} \phi \\
J(f, \phi) & = \pm D\left(\Delta_{1} \phi\right)^{1 / 2}
\end{aligned}
$$

where $J(f, \phi)$ is the Jacobian, and $\Delta_{1} \phi$ is the first order Beltrami differential parameter.

THEOREM. Equation (2) is a necessary and sufficient condition that the family of curves $\phi=$ const. be one family of the net without detours associated with the distance function $f(u, v)$.

If the other family of the net is $\psi=$ const., then

$$
J(f, \psi)= \pm D\left(\Delta_{1} \psi\right)^{1 / 2} .
$$

With the understanding that the square roots may take either sign independently, (2) and (3) may be written

$$
\begin{aligned}
& \phi_{v} f_{u}-\phi_{u} f_{v}=D\left(\Delta_{1} \phi\right)^{1 / 2} \\
& \psi_{v} f_{u}-\psi_{u} f_{v}=D\left(\Delta_{1} \psi\right)^{1 / 2} .
\end{aligned}
$$

Solving for $f_{u}$ and $f_{v}$, we obtain

$$
\begin{aligned}
& f_{u}=\frac{D\left[\phi_{u}\left(\Delta_{1} \psi\right)^{1 / 2}-\psi_{u}\left(\Delta_{1} \phi\right)^{1 / 2}\right]}{J(\phi, \psi)}, \\
& f_{v}=\frac{D\left[\phi_{v}\left(\Delta_{1} \psi\right)^{1 / 2}-\psi_{v}\left(\Delta_{1} \phi\right)^{1 / 2}\right]}{J(\phi, \psi)} .
\end{aligned}
$$

The integrability condition then gives as a necessary and sufficient condition that the two families form a net without detours

$$
\begin{aligned}
\frac{\partial}{\partial v}\left\{\frac{D\left[\phi_{u}\left(\Delta_{1} \psi\right)^{1 / 2}-\psi_{u}\left(\Delta_{1} \phi\right)^{1 / 2}\right]}{J(\phi, \psi)}\right\} & \\
= & \frac{\partial}{\partial u}\left\{\frac{D\left[\phi_{v}\left(\Delta_{1} \psi\right)^{1 / 2}-\psi_{v}\left(\Delta_{1} \phi\right)^{1 / 2}\right]}{J(\phi, \psi)}\right\} .
\end{aligned}
$$

Performing part of the indicated differentiations, and reintroducing the \pm signs, we obtain as the final form of the condition

$$
J\left[\phi, \frac{D\left(\Delta_{1} \psi\right)^{1 / 2}}{J(\phi, \psi)}\right]= \pm J\left[\psi, \frac{D\left(\Delta_{1} \phi\right)^{1 / 2}}{J(\phi, \psi)}\right] .
$$


THEOREM. Equation (4) is a necessary and sufficient condition that the two families $\phi=$ const. and $\psi=$ const. form $a$ net without detours.

The choice of signs corresponds to the choice of orientation of the two families. If both signs hold, that is, if both sides of (4) vanish identically, then any orientation is possible.

If one of the families, say $\phi=$ const., is given in the form $M d u+N d v=0$, then substitution of $M$ and $N$ for $\phi_{u}$ and $\phi_{v}$ in (4) gives a condition applicable to that case, for at no point was use made of the integrability condition for $\phi_{u}$ and $\phi_{v}$.

Equivalent conditions for a net without detours have been given for the plane by Scheffers* and for surfaces by Rothe, $\dagger$ but neither of these writers gives the conditions in the invariant forms (2) and (4).

Dartmouth College

\section{INDICES IN CUBIC FIELDS $\ddagger$}

\section{BY MARSHALL HALL}

1. Introduction. Let $d$ be the discriminant of an algebraic field $K$ generated by a root $\theta$ of an irreducible equation

$$
f(x)=x^{n}+a_{1} x^{n-1}+\cdots+a_{n}=0,
$$

whose coefficients are rational integers. If $d_{\theta}$ is the discriminant of $\theta$, then $d_{\theta}=k_{\theta}{ }^{2} d$, where $k_{\theta}$ is a rational integer called the index of $\theta$. The ring of polynomials in $\theta$ with rational integral coefficients, $P(\theta)$, is a subring of index $k_{\theta}$ of $R(\theta)$, the ring of algebraic integers of $K(\theta)$. In particular, if $k_{\theta}=1, P(\theta)$ is identical with $R(\theta)$. Dedekind $\S$ first showed that it is not always possible to find an integer $\alpha$ in a given field such that $k_{\alpha}=1$, by exhibiting certain fields of third and fourth degree in which there is a divisor common to the index of every integer of the field. Extensive researches have been made as to what these common index divisors may be for fields of a given degree. For $p$ to be a common index divisor of some field of degree $n$, the condition $p<n$

* Leipziger Berichte, vol. 57 (1905), p. 353.

$\dagger$ Berliner Mathematische Sitzungsberichte, vol. 7 (1908), pp. 14-15.

$\ddagger$ Presented to the Society, April 19, 1935.

$\S$ R. Dedekind, Göttinger Gelehrte Anzeigen, 1871, pp. 1481-1494. 